\title{
Para gustos, los colores. El matizado mundo delirante de los géneros: La "patergaynidad" en otro tipo de familia en Azul y no tan rosa de Miguel Ferrari
}

\author{
Pércio Castro \\ University of Dayton \\ pcastro1@udayton.edu
}

\begin{abstract}
Blue and Not So Pink (2012), created by Miguel Ferrari as director and screenwriter, received innumerable positive and negative critiques. In spite of the negative evaluations, the film managed to become a great box office success in Venezuela and in 2013 obtained the Goya Award for the best Spanish American film.

This study will analyze the film's most significant themes; that is, that the consideration of human diversity as an element should be accepted in our societies, the possibility of giving voice to persons who suffer discrimination
\end{abstract}


and the issue of self-acceptance. The notion that the film proposes that there should be other types of families in our societies will be considered: families with gay parents, those with only one parent, and therefore, families that are formed with no blood relationship whatsoever. The trajectory of the main characters within parameters that are juxtaposed and, at the same time, complement each other will be observed-the private and public world, the social sphere and the familial one. Consequently, the transformation that occurs in the family unit to include more unbiased parental rights for homosexuals and transexuals in the formation of a new kind of family core will likewise be examined.

By way of conclusion, it will be observed how the musical diversity of the film is developed intradiegetically to support gender diversity, as well as an examination of the concept of gender within heterocentric society and the way in which relationships of homosexual couples and transgender couples challenge patriarchal society and the dichotomous, binary system it adopted.

Keywords: Latin American Film; Film Studies; Venezuelan Film; LGBTQ Rights; Human Rights; Queer Cinema

\section{Resumen}

El primer largometraje, Azul y no tan rosa (2012), de Miguel Ferrari como director y guionista recibió innumerables críticas positivas y negativas. A pesar de las evaluaciones negativas, la película alcanzó tener un gran éxito de taquilla en Venezuela y obtuvo en 2013 el Goya de mejor película hispanoamericana.

Este estudio analizará la temática de mayor peso de la película; es decir, la de considerar la diversidad humana como un elemento que debe de ser aceptado en nuestras sociedades, la posibilidad de dar voz a seres discriminados y la cuestión de la auto aceptación. Se considerará que la película postula la consideración que deberían de tener otros tipos de familia en nuestras sociedades: familias homoparentales y familias monoparentales, y por ende, familias que no se forman por medio de una relación sanguínea. Se observará la trayectoria de los personajes principales dentro de parámetros que se yuxtaponen y al mismo tiempo se complementan-el mundo privado y el público, la esfera social y 
la familiar. Se estudiará, por lo tanto, la transformación que ocurre en la célula de la familia para incluir derechos parentales más ecuánimes para los homosexuales y transexuales en la formación de un nuevo tipo de seno familiar.

A título de conclusión, se planteará cómo la diversidad musical del film se desarrolla intradiegéticamente para apoyar la diversidad de género y se examinará el concepto de género dentro de la sociedad heterocéntrica y la manera en que las relaciones de parejas homosexuales y el transgenerismo desafían la sociedad patriarcal, el sistema dicotómico y binario adoptado.

Palabras clave: Cine Latinoamericano; Estudios de Cine; Cine venezolano; derechos LGBTQ; Derechos humanos; Cine queer

- I concepto de que "No se nace mujer, se llega a serlo..." de Simone de - Beauvoir generó un gran alboroto en 1949 con la publicación de su libro, El segundo sexo. Hoy, en la sociedad cambiante, tolerante, y multigénero del siglo XXI, se podría ampliar este concepto para abarcar a cualquier ser humano: a los hombres, a las mujeres y al tercer sexo. Marta Lamas, en su artículo “La perspectiva de género”, plantea varios interrogantes: ¿Hay o no hay una relación entre la diferencia biológica y la diferencia sociocultural? ¿Qué posibilidades hay de modificar los papeles sexuales si son determinados biológicamente? ¿Por qué la diferencia sexual implica desigualdad social? Lamas menciona que "lo que el concepto de género ayuda a comprender es que muchas de las cuestiones que pensamos que son atributos 'naturales' de los hombres o de las mujeres, en realidad son características construidas socialmente, que no tienen relación con la biología" (4).

Azul y no tan rosa (2012), el primer largometraje de Miguel Ferrari como director y guionista, trae a la pantalla la temática de la diversidad de género en una sociedad multigénero en mutación que todavía busca encontrar un equilibrio. En este ensayo se estudiará la película de Ferrari como un elemento que confronta los prejuicios sociales de nuestras comunidades revelando, consiguientemente, su hipocresía y el prejuicio de que la heterosexualidad es 
la "normativa obligatoria y aceptada" y que la homosexualidad es la opcional $y$, por ende, rechazada. Se analizará, en particular, la trayectoria de los personajes principales dentro de parámetros que se yuxtaponen y al mismo tiempo se complementan: el mundo privado y el público, la esfera social y la familiar. Se considerará, consecuentemente, la transformación que ocurre en la célula de la familia para incluir derechos parentales más ecuánimes para los homosexuales y transexuales en la formación de un nuevo tipo de seno familiar. Finalmente, se planteará, a título de conclusión, cómo la diversidad musical del film se desarrolla de forma paralela, de manera intradiegética, es decir, dentro de la trama para completar y celebrar la diversidad de género.

A pesar de las varias críticas negativas recibidas, que señalan frecuentemente la falta de profundidad que tiene la trama y el final, quizás, un poco apresurado del argumento, "demasiado previsible y poco inteligente" (Barriga 2), esta producción venezolana-española alcanzó un gran éxito de taquilla en Venezuela, permaneciendo en cartelera durante más de diez semanas, y logrando vender más de seiscientas mil entradas. La cinta recibió en 2013 el Goya a la mejor película hispanoamericana.

Las cuestiones abordadas en Azul y no tan rosa son múltiples y diversas: la homosexualidad, el matrimonio de parejas del mismo sexo, y la homofobia; la violencia doméstica, la violencia de género, y la opresión contra la mujer; la paternidad y las relaciones familiares; la hipocresía social, entre otros temas que incluyen la anorexia y otros trastornos de la conducta. José Barriga, por ejemplo, menciona que Ferrari “deleita la retina del espectador con una estética impresionante y deja de lado profundizar en un argumento que merecía ser explotado" (2) y añade que "[h]ablar de homofobia, incomunicación de padres e hijos, violencia doméstica y tolerancia, no es meramente citar breves casos de estas problemáticas, sino profundizar inteligentemente en los temas y arrogar interrogantes a la mente del espectador" (2). Otros, no obstante, discrepan de esta perspectiva y aseveran que la película "es transformación, 
es atrevimiento brillante, es un beso distinto, un rescate del concepto de la familia, una cachetada a los burlones, una patada en el culo a los odiantes. Y sobre todas las cosas es un mensaje que llega en momentos de necesidad" (Arzola 2).

La temática de mayor peso en el film tal vez sea la de considerar la diversidad humana como un elemento que debe de ser aceptado en nuestras sociedades; la posibilidad de dar voz a seres discriminados y, por ende, la cuestión de la autoaceptación: “[c]oncebí la historia sin el propósito de hacer algo distinto. Simplemente quería escribir una historia que tratara sobre el derecho de cualquier persona a ser y pensar diferente [...] Es una historia sobre el reencuentro. Sobre el respeto que merece cualquier persona a tener una opción diferente [...] Sobre el derecho que tiene cualquier persona a pensar distinto y que no por ello deba ser discriminado" (Ferrari citado en Tavárez 3).

Desde un principio, con la apertura de la película, el espectador puede visualizar parte de la trama de la historia. El director la inaugura con escenas de un ballet moderno en el cual identificamos a seis personas-tres del sexo femenino en ropa interior blanca y tres del sexo masculino en calzoncillos negros-que bailan los unos con los otros mientras uno de los personajes principales, el fotógrafo Diego, fotografía a los bailarines. Ferrari intercala en esta presentación escenas de un parto en el agua en el cual encontramos el otro eje fundamental de la trama, que gira en torno a Fabricio, médico y pareja de Diego. Durante el transcurso del ballet, parece que estas personas, los bailarines, estuvieran buscando su otra mitad dentro de su propia realidad sexual. Así que el baile concluye con la formación de tres parejas distintas: una homosexual gay, una lesbiana y una heterosexual, y culmina con la entrega corporal de cada elemento que forma las parejas a través de un simbólico beso en la boca. Nos presenta Ferrari en este momento a la coreógrafa Delirio del Río, transexual y amiga de Diego. Es quizás por medio de este personaje que el director venezolano logra marcar de forma sutil que su película va más 
allá de la discusión de la temática gay, y busca revelar la complejidad más amplia de una sociedad multigénero, una sociedad que desafía las barreras del binarismo sexual tradicional. O como la propia Delirio se presenta: "En realidad mi nombre es Alejandra. Mi nombre artístico es Delirio, Delirio del Río. Como Dolores, pero Delirio. Claro que antes de llamarme Alejandra, me llamaba Alejo, pero luego me operé y me cambié el nombre [...] Es que era bailarín, pero luego quise ser bailarina. Y mientras estaba en el cambio, no era ni una cosa ni la otra, me llamaban Delirio y así me quedé" (21:17). Dicho en palabras del propio Ferrari: “[n]o es una película de temática gay porque no hay películas de temática hetero. Es una película que trata de lo diferente, de cómo [la diferencia] puede crear escozor en una sociedad que está llena de prejuicios" (Ferrari citado en Ramón 2). El personaje de Delirio en sí encarna distintas posibilidades a nivel de sexo y género. Nació con sexo masculino, se operó más tarde, físicamente, para asumir su identidad femenina como mujer y a pesar de ser coreógrafa, trabaja como si fuera un travesti con sus pelucones, enormes tacones, maquillaje exagerado y un consolador como micrófono en un antro llamado 69 donde interpreta éxitos conocidos- "No soy una señora", y "A punto de caramelo"-de la cantante de origen peruano, Melissa.

Es evidente que el cine de Almodóvar le impactó a Ferrari cuando estudió dirección de cine en España y en "Rueda de prensa de Azul y no tan rosa", el cineasta, hablando del director español, menciona que: "fui a un ciclo de Almodóvar que hicieron en Venezuela y quedé asombrado con sus historias inverosímiles pero que al final te acabas creyendo" (2). No es difícil ver algunas semejanzas y cierta afinidad temática entre Azul y no tan rosa y algunas de las películas de Pedro Almodóvar. Ferrari no niega su influencia; sin embargo, los diálogos de esta cinta venezolana parecen ser más humanos, más parecidos al discurso cotidiano y popular, a los diálogos que encontramos a diario en nuestro propio vivir como personajes de la vida y se distancian, consecuentemente, de los diálogos más abstractos o intelectualizados creados por el director 
manchego, que muchas veces se acercan a las figuras alegóricas o satíricas del teatro del absurdo. Sin embargo, Delirio, como personaje, puede tener cierta afinidad con Tina en La ley del deseo. Ambas son transexuales y los dos personajes encarnan el tema de la diversidad e insertan en la trama la idea de que el erotismo y la sexualidad no tienen sexo; que el "género" transciende al sexo y que los sentimientos de amor, soledad, rechazo, tristeza y abandono pueden ser sentidos por cualquier ser humano: “[q]uise hablar no solo de la homosexualidad, sino de la diversidad, de lo que es considerado diferente: un homosexual, un transexual, un chico que se siente feo porque no corresponde al patrón de lo que es considerado belleza, y una chica que es discriminada solo por ser mujer" (Ferrari citado en Ramón 3). En resumen, a través de estos personajes, Ferrari da voz a distintas personas y puntos de vista de la sociedad y revela la complejidad de diversos problemas con los que se enfrentan los individuos de una sociedad multigénero.

Almodóvar ha mencionado en diversas ocasiones que La ley del deseo no trata de la homosexualidad, sino del amor en general y de la familia (Smith 190). De igual manera, lo mismo puede ser dicho sobre Azul y no tan rosa, como señala su director: "[e]I tema central de Azul y no tan rosa gira en torno a la relación de un padre y un hijo que se reencuentran y se ven obligados a solucionar sus diferencias. Es una historia sobre el amor, la amistad, sobre la familia en su sentido más amplio" (Tavárez 3). La película de hecho observa el concepto de familia desde distintos enfoques. Es decir, va más allá de los arcaicos parámetros demarcados por la sociedad patriarcal y heteronormativa de lo que debe constituir o ser una familia. Dicho de otra forma, rompe con las dogmáticas creencias de la sociedad androcéntrica para plantear un nuevo modelo de familia a lo largo del film. Así pues, la película presenta varias posibilidades de lo que es y lo que podría ser considerado "familia" en el siglo XXI, sin eliminar por ello la tríada tradicional familiar del padre, la madre y los hijos, estructura de roles tradicionales visibles en parte en las 
familias de Diego y de cierta manera también en la de Fabricio. Es decir, contar con miembros familiares con distintos tipos de orientación sexual no impide que estas familias tengan los mismos problemas que cualquier otra, con sus prejuicios, con sus desacuerdos, con sus alegrías y tristezas. La película a través de esta temática, postula así la consideración y el lugar que deberían de tener otros tipos de familia en nuestras sociedades: familias homoparentales y familias monoparentales $y$, por ende, familias que no necesariamente se forman por medio de una relación sanguínea. Quizás, incluso, la temática de la película podría romper con sus propios parámetros para que, como sociedad, empezáramos a plantear los derechos de paternidad de los homosexuales no sólo los derechos biológicos, sino los de adopción o cualquier otra posibilidad y pudiéramos entonces establecer derechos a los que definiremos aquí como derechos a la "patergaynidad" o a la "parentalidad" gay ya que "[l]o más frecuente en los países democráticos es que la paternidad biológica esté protegida, aún a título individual, mientras que la paternidad compartida por una pareja esté reservada al matrimonio heterosexual, mientras que las parejas homosexuales están excluidas de esta opción" (Montes 2). Este horizonte legislativo consideraría no solamente los derechos de grupos individuales (mujeres, gays, lesbianas, transexuales, etc.), sino que permitiría desarrollar un sistema que respaldara una noción de igualdad todavía más abarcadora basada en la justicia de género. En este sentido, Azul y no tan rosa se vuelve parte de un grupo de filmes que reclaman esta justicia. Ferrari en una de sus entrevistas, al hablar sobre el tema de la homosexualidad, afirma que "hasta esta película nunca dos hombres se habían besado en una película venezolana [...] Es un tema latente [...] Hay una necesidad de mostrarlo" ("Rueda de prensa" 2).

Es evidente que Diego, en lo que corresponde a la paternidad, no se encuentra con este tipo de dilema en la película puesto que Armando es su hijo biológico aunque viva en España con su madre biológica. Sin embargo, 
especialmente en la época del estreno de la película, en un país como Venezuela-, al igual que en otros países latinoamericanos, en los cuales los homosexuales continuaban siendo discriminados, donde ni siquiera el matrimonio entre personas del mismo sexo era considerado-aunque Diego y Fabricio hubieran podido llevar a cabo la formación de "una pareja como otra cualquiera", como lo mencionó Fabricio, y si quisieran adoptar o tener un hijo, solo uno de ellos podría legalmente ser el padre del niño. Dicho esto, la familia como célula social, no se encuentra en crisis, sino que, a partir del momento en que empezamos a revalorar y a redibujar las convenciones y a desmantelar el patriarcal androcentrismo de la sociedad, se evidencia una deconstrucción de los modelos tradicionalmente asociados con la paternidad y con la maternidad, desestructurando, por ende, el binarismo femenino y masculino.

Azul y no tan rosa no solo proporciona un concepto más profundo de lo que podríamos determinar cómo familia en el siglo XXI, sino que también plantea a la audiencia una nueva visión de la salida del clóset. Es innegable que ni Diego ni Fabricio esconden su homosexualidad y ninguno de los dos es presentado con las estereotipadas características gay de "hombres amanerados o con cuerpos de gimnasio o promiscuos. [Ferrari se aleja de estos prototipos para] mostrar seres humanos que sienten y padecen como cualquier otro" (Ferrari citado en Tavárez 3-4). Con la llegada de Armando a Caracas para vivir con su padre después de una ausencia de cinco años, ambos tienen que reencontrarse como personajes que son partícipes de una historia en común, la de ser padre e hijo. Diego no disimula su homosexualidad; no obstante, el tener que exponer su orientación sexual a su hijo adolescente y heterosexual le da al público una práctica alternativa a la convencional estructura de la salida del clóset. En este caso, no es el hijo sino el padre quien tiene que hacerlo. En ningún momento Armando parece criticar la homosexualidad de su padre y la única vez que lo hace, al poner una película homosexual en la tele, lo hace 
no para atacar directamente a su padre, sino para reclamar su ausencia y el abandono que le fue impuesto por la deserción paterna:

-Apaga eso.

— ¿Qué pasa? ¿Te da vergüenza que sepa que eres maricón?

-Apaga eso.

-Porque eres maricón, ¿no?

$[\ldots]$

— ¡Escúchame! Sí, soy maricón y también soy tu papá y contigo me he mostrado tal cual soy sin estar escondiendo nada.

$[\ldots]$

— ¿Qué tengo que entender, papá? ¿Que pasas de mí? ¿Es eso lo que tengo que entender? (37:04)

Cuando Armando descubre que la pareja de su padre ha sufrido una paliza homófoba y que se encuentra hospitalizado y en estado de coma, apoya a Diego y le brinda también apoyo cuando Fabricio fallece. La homofobia se representa de manera explícita en la película, haciendo patentes los prejuicios sociales a través de un programa televisivo bastante estereotipado de temas variados al estilo "talk show" que parece ser uno de los programas favoritos de la sociedad venezolana en la película. La primera vez que aparece la emisión televisiva es en la casa de los padres de Diego donde se encuentran no solamente sus padres, sino también su hermano, su cuñada y su sobrino. Estrellita, la presentadora, anuncia el tema del día que abordará el programa: “El matrimonio de gays y lesbianas. ¿Se acerca el fin del mundo?” El niño le pregunta al abuelo qué es gay y el abuelo le contesta: "un maricón” (12:59). Más tarde, el niño le pregunta al abuelo qué es lesbiana y este le contesta: "una puta camionera" (13:06). Las conversaciones sobre el matrimonio del mismo sexo, la homosexualidad, y la diversidad de géneros siguen en el seno familiar con distintos comentarios, enseñándonos la falta de conocimientos sobre la temática abordada, los prejuicios homófobos y la curiosidad que podríamos 
encontrar en cualquier familia: "un matrimonio de verdad es el mío con vuestra madre y no uno de estos modernos de ahora" (13:50) [...] "No es por nada, pero yo prefiero que mi hijo sea un delincuente que sea un maricón” (13:58). En un momento dado, Patricia, la cuñada, le pregunta a Diego sobre su amigo que es médico y le menciona que le parece raro que con esa edad todavía no se haya casado. Diego le contesta que Fabricio está a punto de casarse y cuando Patricia indaga con quién, este contesta simplemente que con él. El sobrino entonces le pregunta: “Tío, ¿eres maricón?" (15:18). Momento en que la madre de Diego interrumpe la conversación, tirando el postre sobre la mesa y preguntando si alguien lo quiere. En resumen, a partir del momento en que la cuestión "gay" interfiere con el orbe privado de la familia tradicional, se transforma de alguna forma en tabú, puesto que “ide eso no se habla!” Dicho de otra forma, "Ferrari's film explores the transnationality of LGBTQ issues and how they operate in the Spanish-speaking world... and relies more on the dialogues and interactions between characters to explore hard-hitting themes such as homophobia, hate crimes, and body acceptance... [bringing] to light important queer issues in Venezuela" (Venkatesh 2).

Ferrari parece sumar la temática de su film a otras películas iberoamericanas que también tratan los prejuicios y el acoso a los homosexuales tanto al nivel público social como al nivel privado familiar. En el largometraje peruano de Francisco Lombardi, No se lo digas a nadie (1994), el padre del protagonista también menciona que prefiere tener un hijo retrasado mentalmente antes que un hijo "maricón".

La homofobia en Azul y no tan rosa se hace todavía más evidente con el desarrollo de la historia, y no solo por la horrenda paliza que recibe Fabricio debido a su orientación sexual, sino dentro del ámbito familiar. El padre de Fabricio le niega a Diego el permiso de entrar a la habitación donde éste se haya hospitalizado. En este momento, la película trae a la pantalla el dilema de muchas parejas homosexuales que sin tener el respaldo legal se 
ven completamente paralizadas y sin derechos ante un sistema médico que reconoce solamente las tradicionales relaciones familiares heteronormativas:

-Voy a verlo.

—Lo siento, Diego. Mi marido no quiere que entre nadie. Solo él y yo.

- ¿Qué?

-Que no quiero que lo veas ni tú, ni los depravados con los que andas.

-Yo no ando con depravados y lo voy a ver aunque usted no quiera.

-Tú no eres nada de mi hijo. Tú tienes la culpa de todo lo que le pasó.

-Fabricio y yo somos pareja y apenas él salga de aquí, nos vamos a ir a vivir juntos.

-Antes prefiero que se muera.

—Usted es igual que los hijos de puta que jodieron a Fabricio, igual. (34:32)

Diego maniobra para lograr entrar en la habitación donde está Fabricio y para decirle que esperará por él todo el tiempo que haga falta. Antes de salir de la habitación saca dos anillos de compromiso y le dice a Fabricio: "te traje esto para que no se te olvide. Te lo iba a dar aquella noche, pero tú no llegaste" (42:21). Ferrari trabaja la escena con maestría, con distintos planos, eliminando el monólogo de Diego, introduciendo el silencio y subiendo el volumen de la música extradiegética que acompaña la escena. A través de tomas en primer plano y plano detalle, Diego le pone el anillo en el dedo a Fabricio y el otro en su propio dedo y sobrepone una mano sobre la otra. Se concreta en esta secuencia de escenas una boda simbólica espiritual que clama y reclama el derecho al matrimonio de parejas del mismo sexo, retando, por consiguiente, la falta de reconocimiento y legislación sobre el tema en Venezuela y en otros países. La despedida se concluye cuando, también en primer plano, Diego le da un beso en la frente a Fabricio mientras una lágrima se escurre por su mejilla.

Ya en el título de la película, Azul y no tan rosa, se demarca claramente la asociación cromática supuestamente estable de los distintos géneros: azul para los varones y rosado para las hembras. Dicho de otra forma, el título 
del film alude a la división binaria cultural, histórica y socialmente adquirida que estipula ciertos criterios y colores según el sexo del bebé, muchas veces a través del proceso de asignación de género, basado simplemente en los genitales, y que ocurre mayormente durante el nacimiento y muchas veces, hoy, incluso antes del nacimiento por medio de ultrasonografías. Claro está que podríamos interpretar el título de forma más metafórica, considerando que la vida no siempre es color de rosa, pensando en las batallas que cada uno de los personajes principales tiene que afrontar en su vivir diario. No obstante, es evidente que las relaciones de parejas homosexuales y el transgenerismo de los personajes protagonistas y de las personas en general desafían la sociedad patriarcal, el sistema dicotómico y binario adoptado que, a su vez, se compone de roles y actitudes que enfatizan ciertas características como femeninas y otras como masculinas y desembocan en la yuxtaposición del paradigma del machismo y del marianismo; es decir, una sociedad que acentúa ciertos roles y funciones como masculinos y otros como femeninos: "[e]ntre los autores que estudian género y sexualidad hay consenso en entender al [sic] género como una construcción cultural que, a partir de la diferencia sexual, establece qué características definen a la mujer o al hombre en una sociedad determinada. Para la sociedad patriarcal los diferentes roles sexuales surgen de la división del trabajo, la que, a su vez, se basa en la diferencia biológica” (Díaz Rodríguez 3).

El término "género" puede provocar cierta confusión ya que ha sido definido y redefinido por estudiosos del campo desde la década de los cincuenta. Para hablar de género en español es necesario que nos adueñemos del concepto anglosajón y de la terminología que poco a poco es adoptada en nuestros usos lingüísticos para poder abarcar ideas y conceptos que vayan más allá de la sencilla división entre masculino y femenino. En 1955 John Moley, analizando las conductas relacionadas con los comportamientos de hombres y mujeres, propuso el término "rol de género". En 1968 Robert Stoller definió lo que se conoce psicológicamente como “identidad de género" y aclaró que esta no se relaciona 
de manera mecánica con el sexo biológico. El movimiento feminista de los años 70 enfatizó que las desigualdades entre hombres y mujeres son construcciones sociales y no biológicas (Murguialday 1). Las muchas teorías acerca de cómo se define esta identidad de género siguen siendo estudiadas y desarrolladas a través de las décadas en búsqueda de un común denominador que quizás pueda explicar lo que es el género y de qué forma se adquiere la identidad de género. Seguimos a lo largo de los años acoplando nuevas nomenclaturas y concepciones al término "género" para definir las relaciones, orientaciones y formaciones sexuales de los seres humanos: homo-bi-pan-trans-inter-antrosexual. Desafortunadamente, la sociedad sigue desarrollando de forma paralela y reactiva distintas fobias: homo-lesbo-transexo-fobia. Según Tania Esmeralda Rocha Sánchez conceptuar lo que pueda ser la identidad de género ha sido una tarea difícil, sobre todo por la confusión que rodea al término "género":

De acuerdo con Hawkesworth (1977) este término en principio tiene al menos 25 usos diferentes... [E]n algunos se hace evidente la noción de identidad: (a) Sexo: Diferenciación biológica; (b) Sexualidad: prácticas sexuales y conducta erótica; (c) Identidad sexual: designación de un individuo como heterosexual, homosexual, gay, lesbiana, bisexual, transexual, o asexual; (d) Identidad de género: sentido psicológico de sí mismo como hombre o mujer; (e) Rol de género: un conjunto de expectativas culturales específicas acerca de qué es apropiado para un hombre y para una mujer; (f) Identidad de rol de género: grado en el cual una persona aprueba y participa de un conjunto de sentimientos y conductas consideradas como apropiadas para sí mismo en su género constituido culturalmente... (3)

Aunque intentemos encontrar una posible y, tal vez, más lógica concepción para la pluralidad de las distintas identidades de género disponibles, las fobias se desarrollan como defensa contra los retos al sistema heterocéntrico y los patrones heteronormativos histórica, cultural, social y políticamente aprendidos. Es decir, si la identidad de género no amenaza la preservación del 
poder patriarcal androcéntrico, la sociedad no busca una razón para rechazarla. Consecuentemente, es fácil percibir que se hable de tantas fobias; no obstante, no es sorprendente que no escuchemos hablar del término "asexofobia."

En Azul y no tan rosa, la formación de la nueva familia se constituye con distintos elementos, generando una familia multigénero: un homosexual como padre, Diego; una transexual como madre, Delirio; un adolescente heterosexual con un posible trastorno dismórfico corporal como hijo, Armando; y una mujer adulta heterosexual embarazada como hermana, Perla Marina; esta última, separada de su pareja a causa de la violencia doméstica, de la violencia de género. La nueva familia se apoya mutuamente tanto en los momentos difíciles como en las circunstancias favorables. La familia brinda apoyo a Diego para que pueda viajar a Mérida y plantar un pino en homenaje a Fabricio. Le ayuda a Armando a aprender a bailar el tango para que pueda conocer e impresionar a una novia cibernética y sentirse más seguro de sí mismo. Le apoya a Delirio en sus espectáculos para que pueda decir adiós a Luis Fernando-su primer amor como Alejo-y, consecuentemente, decir adiós a su mitad masculina del pasado. Y, finalmente, ampara a Perla Marina durante la separación para que pueda creer en sí misma como mujer independiente.

De la misma forma que la película ofrece una multiplicidad de alternativas en lo que concierne al género humano y su diversidad, su director intercala en el film también una multiplicidad de armonías musicales: desde la música pop de los años 80 con Melissa, "No soy una señora” y “A punto de caramelo", hasta el tango "Poema" de 1925 de la orquesta de Francisco Canaro con música de Mario Melfi, letra de Eduardo Bianco y cantada por Roberto Maida; desde un aria de Vicenzo Bellini, "Casta Diva”, de la ópera Norma hasta la música folclórica "Tonada de luna llena" del compositor y poeta venezolano, Simón Díaz. La combinación de música y silencios utilizada por el cineasta conduce a la audiencia a vivir junto a los personajes sus angustias y sus alegrías. Tanto en el plano diegético como en el extradiegético, la música transporta a los 
espectadores a través del tiempo fílmico, a través de los días, y les lleva a compartir el torbellino de emociones de todos los protagonistas y sus sentimientos más recónditos. El tango "Poema” le sirve al cineasta de telón de fondo para la trama (Ferrari citado en Tavárez 4) y de la misma forma en que viven los protagonistas la letra del tango- "fue el poema de ayer / que yo soñé / de dorado color, / vanas quimeras del corazón”-, Ferrari escribe su propio poema fílmico y compone su propio tango poema al montar el rompecabezas de la vida de sus personajes. La heterogénea banda musical de la película parece interpelar a la audiencia planteándole si al aceptar esta gran variedad de géneros musicales no sería lógico también ser tolerante con la diversidad sexual: ¿por qué no podemos aceptar de la misma forma nuestras múltiples y distintas identidades de género como sociedad?

La película también ha sido criticada por ser a veces un poco melodramática y exagerada como si fuera una telenovela, casi un culebrón. Su director menciona que siempre defiende las telenovelas: “A los venezolanos les puede quitar el pan, pero no las telenovelas... El melodrama no puede ser visto como algo menor..." ("Rueda de Prensa" 2). Si observamos cuidadosamente, nuestras vidas, la vida de nuestras comunidades, nuestra diversidad de género, la vida de nosotros, espectadores, no es tan distinta de las vidas de estos personajes. Nuestro existir también podría ser parte de una telenovela. O como lo dice Perla Marina: “Ah pues, bueno con mi vida podría hacerse sino un culebrón, sino una boa constrictor de mil capítulos" (39:23).

Al final Azul y no tan rosa vuelve a los colores del título. Perla Marina está a punto de tener el bebé y está arreglando las cosas en el cuarto para su llegada cuando su madre le critica los colores seleccionados: “y resulta si tienes un varón no le va a poner escarpines rosados. Ah mamá, no sé quién inventó esto de que el azul es para los varones y el rosa para las hembras. A mí me gustan los dos colores [...] Yo lo voy a querer igual, sea hembra o varón. ¿Cuál es la diferencia?” (1:44:20) La pregunta se queda en el aire, como una 
pregunta retórica que busca una respuesta dentro de una sociedad disímil. Perla Marina de hecho tiene un varón y lo nombra Fabricio, así que sabemos que es del sexo masculino, pero no sabemos con qué género se identificará. La película cierra así su ciclo con escenas de muerte y vida, con otro parto en el agua. El programa de Estrellita es reemplazado por "Noches de delirio". Y la presentadora ahora, Delirio del Río, "una mujer con un par de tacones" (1:48:05) como afirma Perla Marina, anuncia el tema de su programa: "Hoy en 'Noches de delirio,' un programa sobre la diversidad: soy como soy, ¿y qué?" (1:48:48) Un programa que recalca que "vivimos en una sociedad plural donde todos tenemos cabida y donde todos debemos ser escuchados" (1:48:30).

En conclusión, con Azul y no tan rosa, Ferrari desafía los cánones binarios de la sociedad androcéntrica que determina indiscriminadamente lo que es masculino y femenino para exponer la falta de derechos y la desigualdad sexual dentro de un contexto social. A través de la historia de sus protagonistas reclama los derechos de la "parentalidad" al nivel homo o monoparental y trae a la superficie la idea de que el concepto de género no tienen una relación directa con la biología. Al insertar una diversidad de géneros musicales el cineasta parece sutilmente proponer que si podemos aceptar esta diversidad musical, deberíamos, por ende, poder acoger también la diversidad multigénero del ser humano del siglo XXI. En otras palabras, con Azul y no tan rosa, Ferrari compone su propio tango; pinta un cuadro de escenas, a través de la música, a través de los dramas de sus personajes; pinta un lienzo dentro del matizado mundo de los géneros con tintes rosados y azulados y a veces distintos tonos de gris. Nos lleva a reflexionar sobre nuestros propios prejuicios y deliberar como audiencia partícipe: quiénes somos, quiénes son las personas a las cuales queremos, con las cuales compartimos nuestras vidas, con las cuales formamos nuestras familias; quiénes son los personajes secundarios, los protagonistas y antagonistas de nuestro propio culebrón, la vida, que muchas veces es azul y no tan rosa. 


\section{Obras citadas}

Arzola, Daniel. “Azul y no tan rosa y cien colores más”. Blogspot, 4 Dec. 2012, arzolad. blogspot.com/2012/12/azul-y-no-tan-rosa-y-cien-colores-mas.html. Accessed 14 Feb. 2015.

Azul y no tan rosa. Directed by Miguel Ferrari, TLA Releasing, 2013, DVD.

Barriga, José. "Critica Azul y no tan rosa". Cine 2012, Críticas 2012, http:// academyawards2009.blogspot.com/2012/12/critica-azul-y-no-tan-rosa.html. Accessed 14 Feb. 2015.

Beauvoir, S. de. El segundo sexo. Debolsillo, 2007.

Díaz Rodríguez, Alba. “Educación y género”. Colección Pedagógica Universitaria, vol. 40, julio-diciembre 2003, www.uv.mx/cpue/coleccion/N 40/Eb\%20alba\%20 diaz\%20genero\%20educaci\%C3\%B3n.pdf. Accessed 8 Sep. 2020.

La ley del deseo. Directed by Pedro Almodóvar, El Deseo SA, 1987, VHS.

Lamas, Marta. “La perspectiva de género". La Tarea: Revista de Educación y Cultura, diciembre 1995, Guadalajara, México. Accessed 5 Feb. 2015.

Murguialday, Clara. "Género". Diccionario de Acción Humanitaria y Cooperación al Desarrollo, 2005-06. Accessed 5 Feb. 2015.

No se lo digas a nadie. Directed by Francisco Lombardi, Picture This! Entertainment, 2000, DVD.

Montes, Jaime. "Padres que son ellos mismos gays o lesbianas". Ensayos sobre homosexualidad, 2008, http://www.arrakis.es/jmn/Libro/paternidad.html. Accessed 5 Feb. 2015.

"Poema". Lyrics by Eduardo Bianco, music by Mario Melfi, recorded by the Orquesta de Francisco Canaro, interpreted by Roberto Maida, 1925.

Ramón, Esteban. "Azul y no tan rosa, la película que ha sacado al cine venezolano del armario”. RTVE.es, 21 Apr. 2014, www.rtve.es/noticias/20140421/azul-no-tan-rosapelicula-sacado-cine-venezolano-del-armario/923701.shtml. Accessed 5 Feb. 2015.

Rocha Sánchez, Tania Esmeralda. “Desarrollo de la identidad de género desde una perspectiva psico-socio-cultural: un recorrido conceptual”. Interamerican Journal of Psychology, vol. 43, no. 2, agosto 2009. Accessed 5 Feb. 2015. 
"Rueda de prensa de Azul y no tan rosa". Cine Maldito, 24 Apr. 2014, www. cinemaldito.com/rueda-de-prensa-de-azul-y-no-tan-rosa/. Accessed 8 Feb. 2015.

Smith, Paul Julian. Desire Unlimited: The Cinema of Pedro Almodóvar. Verso, 1994.

Tavárez, Felicia. "Entrevista, Miguel Ferrari, director de Azul y no tan rosa". El Antepenúltimo Mohicano: Revista Independiente de Actualidad y Análisis Cinematográfico, 17 Apr. 2014. Accessed 8 Feb. 2015.

Venkatesh, Vinodh. "Top 9 Latin American Films". University of Texas Press, 26 Sept. 2016, utpressnews.blogspot.com/2016/09/top-9-queer-latin-american-films. html\#sthash.IBopAvOO.dpbs. Accessed 15 Apr. 2020. 